\title{
P145 Central Hemodynamic Response to Lower Body Negative Pressure in Individuals with Down Syndrome
}

\author{
Thessa I ${ }^{1, *}$, Sang Ouk Wee ${ }^{2}$, Georgios Grigoriadis ${ }^{1}$, Elizabeth Schroeder ${ }^{1}$, Tracy Baynard ${ }^{1}$, Bo Fernhall ${ }^{1}$ \\ ${ }^{1}$ University of Illinois at Chicago, Chicago, USA \\ ${ }^{2}$ California State University San Bernardino, San Bernardino, CA
}

\section{ABSTRACT}

Introduction: Individuals with Down syndrome (DS) have autonomic dysfunction, which impacts heart rate and blood pressure regulation. Initial evidence also suggests an impaired ability to peripherally vasoconstrict during lower body negative pressure (LBNP). Given the relationship between vessel caliber and wave reflection, this may alter central hemodynamics and hemodynamic load. We aimed to investigate aortic hemodynamics and wave reflection during LBNP in individuals with DS and controls.

Methods: Radial applanation tonometry was performed on 15 individuals with DS (male $n=12$, age $24 \pm 1$ yrs, BMI $29.4 \pm 2.0$ $\mathrm{kg} / \mathrm{m}^{2}$ ) and 21 control participants (male $n=12$, age $24 \pm 1 \mathrm{yrs}$, BMI $24.4 \pm 1.0 \mathrm{~kg} / \mathrm{m}^{2}$ ) before, during and after a $5-\mathrm{min}$ LBNP stimulus of $-20 \mathrm{mmHg}$. Waveforms were calibrated to mean and diastolic brachial pressures. Generalized transfer function was used to estimate aortic pressures (systolic aSBP, diastolic aDBP and mean pressure aMAP), augmentation index (Aix), augmentation index normalized for HR (Aix@HR75), augmentation pressure (AP), reflection index (Rix) and forward (Pf) and reflected wave magnitude $(\mathrm{Pb})$.

Results: Interaction effects were observed for AP, Aix, AIX@75, Rix and Pb $(p<0.05)$ in which DS had reductions in the outcome during LBNP whereas controls had little or no change. Although trending towards a larger drop in aSBP in individuals with DS, no significant interaction effects were found for aSBP, aDBP, aMAP, HR or Pf in response to LBNP.

Conclusion: These results show individuals with DS exhibited greater reductions in aortic pressure waveform characteristics and wave reflections in response to LBNP, indicative of reduced sympathetic control resulting in less peripheral vasoconstriction.

(C) 2019 Association for Research into Arterial Structure and Physiology. Publishing services by Atlantis Press International B.V. This is an open access article distributed under the CC BY-NC 4.0 license (http://creativecommons.org/licenses/by-nc/4.0/). 